\title{
A Service-Oriented Communication Architecture of Graphics Asset Management System
}

\author{
Zi-jian Wang \\ School of Computer Science and Technology, \\ Beijing Institute of Technology \\ Beijing, 100081,China \\ 15063383@qq.com
}

\begin{abstract}
Database technology is of great variety, structure diversity, solving data integration and exchanges sharing of the heterogeneous database system have became the important problem in all fields. Because of XML is the very important data exchange technology to realize data exchange and transmission ability, this paper mainly explores the data exchange problem how to use XML in heterogeneous database system, , and .Net platform have good support to XML and heterogeneous database system, so the combination of XML and.net, can realize data exchange of heterogeneous database system.
\end{abstract}

Keywords-component heterogeneous database; data exchange technique; XML

\section{INTRODUCTION}

Graphics Asset Management System creates a resourcessharing application environment that is emerged by rapid locating huge amount of graphics media resources, which is one of the most pressing requirements used by producers or visual effects designers in media industry. Providing and deploying new principles, solutions and applicant services of media asset management to quickly adapt to the business demands in fickle market is the core concept in the system. After years of improvement and innovations, the graphics asset management system established by SOA has not only become an actual system that keeps abreast with the media business development, but has also been considered as the core competence in the future.

As an advanced architecture model, SOA implement deployment, combination and application of loosing coupling coarse-grained components to provide different web services in the distributed networking system. Servicelayer is the base of SOA, which is able to be directly invoked so that it can effectively trace and control the anthropogenic dependency when the agent communicates with system.

The key concept of SOA is "service", which constitutes provider and consumer. Service provider gets a set of work done, and delivers them to the consumer. The result usually changes the state of user, but also itself, or both. The communication between multiple services might be merely data transmission, or activity coordination among themselves. All services need the same protocol and interaction pattern to realize communication. The "service" means precise definition, complete encapsulation and a function independent of the rest parts in the system. There are several key features of SOA: multilevel coarse-grained, loosing coupling structure, communication through simple and precise Custom Interface among the services, and no reference to underlying programming and communication model.

\section{RELATED WORK}

The new type of digital asset management system consists of audio asset, video asset and graphics asset management braches. The system is designed for handling complex business events because it is faced with more roles and practitioners in media industry, and also adjusts the processing workflows due to operation changes. The whole system must be constructed and maintained in the unified architecture.

According to the requirements above, the author has issued the heuristic system architecture based on SOA. The distributed architecture, as the basis of information transmission extended framework, focuses on the problems of data storage from different types but parallel tasks, complex business-processing rules and online increment and adjustment modules when changes are needed.

\section{EXPERIMENTAL ANALYSIS AND CONCLUSION}

Some documents indicate graphics asset management system as SOA, which is to relate design and realization, based on the specified platform, while some do not. The author supposes that the SOA system, which is not related to any standard, technique or application platform, is able to be taken as reference architecture, thus further proposes that frames that are related can only act as an application of that reference architecture.

The architecture the author designed as an abstract framework that guides to construct the communication and application of graphics asset management system and describes the constitution facilities in communication structure and their relationships without engagement.

1. Design logic and principle

In the article, the distributed architecture is designed as three-tier structure model to separate processing task scheduling layer, operation processing layer and handling executing layer. Using the structure each layer can concentrate on processing specific functions and take its advantage. Extended communication for consumer interaction with multiple protocols and 
policies in the architecture can be implemented in task scheduling layer, workflow customization and executor invocation is built in the operation processing layer, and all the actual function units in handling execution layer that separated on three-tier structure that includes controller, device and handling service module. According to the requirement features such as parallel graphics rendering by GPU, devices divide and deploy by different types but in the unified controller managed and each provides at least one handling module for online functions extended. The System has the ability of parallel task processing, workflow customizing and flexible function extending in complex environment by establishing the architecture as shown below.

In the architecture, task scheduling layer provides interface groups to surrounding consumers. Operation processing layer devises asynchronous scheduling and transaction mechanism to choose and invoke the executors that spread over handling executing layer.

2. Communication architecture design

The architecture based on flexibly and loosely distributed structure needs stable networking infrastructure because all units are spread over different physical hosts. The business task scheduling controller, workflow operation controller and execution node as dominant communication performer between the stages of processing in framework provide reliable communication control. During the transmitting process, messages as the minimum data unit are encapsulated and transferred.

The structure of communication networking

Resource preparation and data execution are two phases in the data communication process. The destination of resource preparation is creating the business workflow instance and integrated data path chain. In contrast, the destination of data execution is ensuring the task data in each executor node analyzed and properly handled when process instance that defined in resource preparation phase flows. "BisManager”, "OperManager" and "ExecNode" are defined as kernel provider for requesting and responding messages in the architecture. BisManager provider:

BisManager directs the affairs of business data communication between the providers and executors. When the task comes out, it creates and maintains an instance from communication tree to ensure the stable connectivity relation. Any messages from the tasks are dispatched and processed via the provider. The activities are mainly for analyzing task messages, projecting and ferrying the processing task to executor by its business type. Using this policy would concentrate on fetching and processing data messages but liberate the construction and maintenance of business communication tree in the rest.

There are three aspects of main duties of BisManager that indicate the following diagram:
$>$ Create, maintain and dispose all the instance of workflow that from the communication tree.

$>$ Keep alive with OperManager provider for asking which executor could be used for the instance.

$>$ Trace and manage the process of workflow, provide the metadata of next node.

OperManager provider:

OperManager hardly participates in the communication that related to the business, but just insures to assign the proper devices from handling executing layer. The activities of OperManager primarily focus on device information interaction, in order to invoke rapid response executors when the tasks to be handled from BisManager request the node and to implement a simple approach to scalability.

ExecNode provider:

ExecNode is the host of cladding service, and is designed to segregate the communication of physical device and executive function. The processed metadata and transaction data of BisManager, OperManager and executors deployed in physical device are transmitted by ExecNode provider to improve the implementation. Each specified executor has a unique identity that registered in ExecNode. When the request has been received, an instance would be created in ExecNode and queued in job list to be performed. When the work is done, ExecNode would notice the BisManager change workflow statement, ask the next node identity from OperNode, then put the result business data and metadata to next ExecNode available, finally, ExecNode notices the OperNode to update its process statement.

3. Transmission relationship of communication

Communication service units (CSU) as the basis of architecture, is the center of kernel communication unit, composed with message processing unit, exception processing unit, state-monitoring unit, database manipulation unit, authentication unit and management unit.

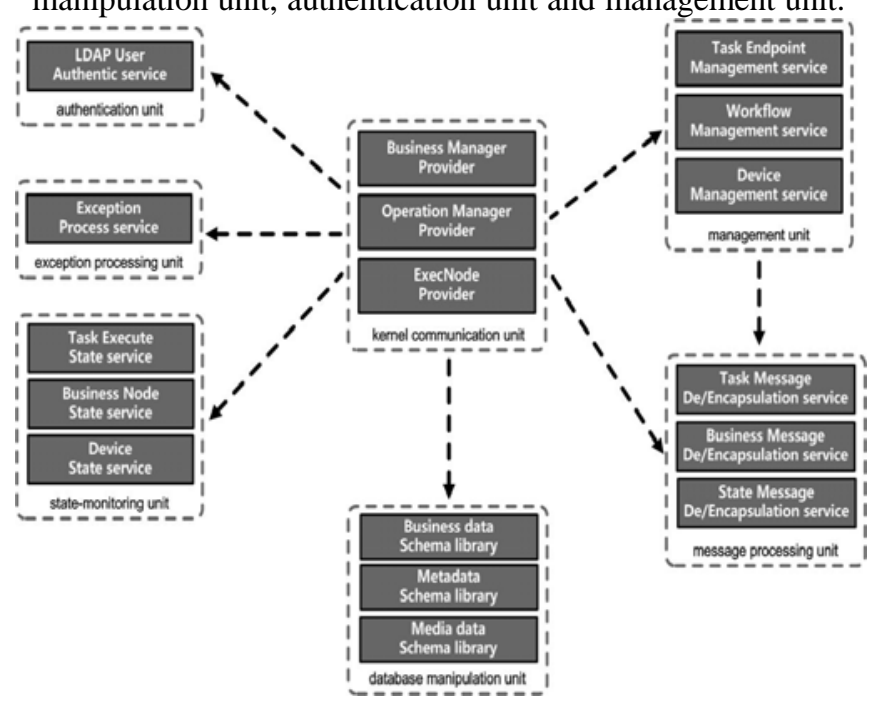


Kernel communication unit has the BisManager OperManager and ExecNode endpoints set responsible for implementing the principle of architecture. To encapsulate or de-encapsulate the messages generated from workflow instance could be processed by message processing unit. According to the type of processing, it can be divided by task message, operation message and statement message. When the exception just as informal formatted or the execution of process occurred, exception processing unit intercepts the message type to process.

BisManager is designed as the resident provider to perform as below:

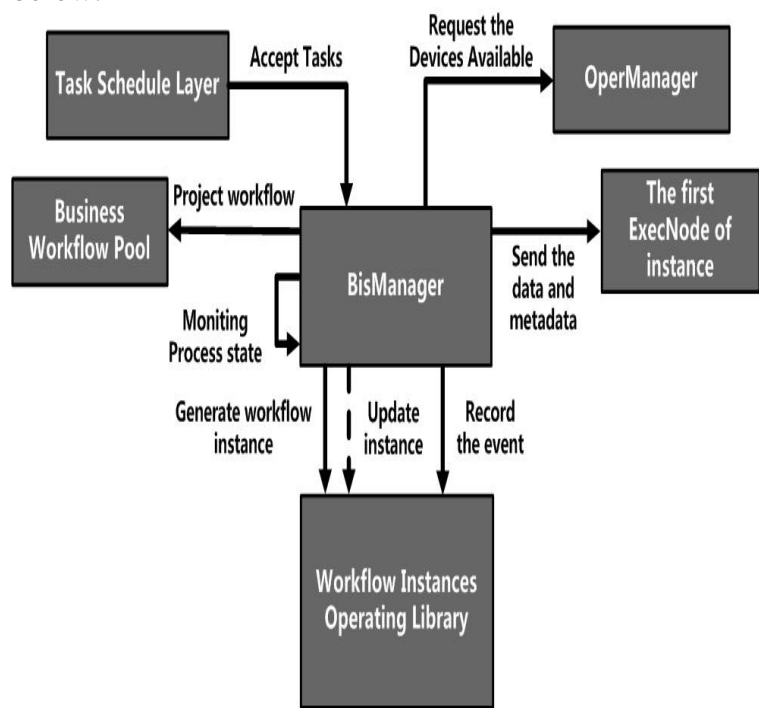

$>$ Respond to the consumer task request;

$>$ Query and project workflow by the task type;

$>$ Generate the project workflow instance;

$>$ Request OperManager the device available;

$>$ Send the business data and executive metadata to the first ExecNode in executor;

OperManager is designed as the resident provider to perform as below:

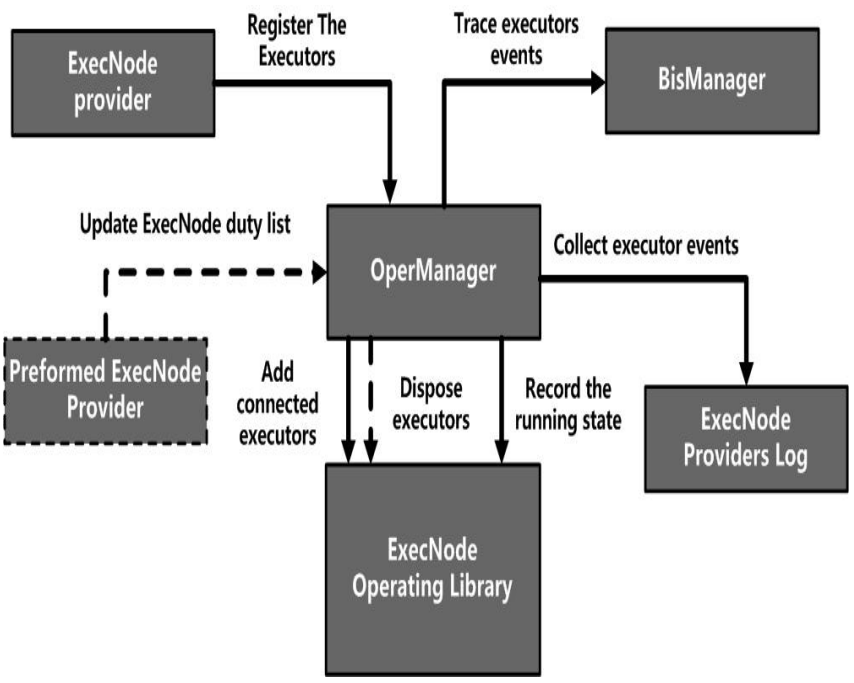

Response the available executor list to the workflow instance;

$>$ Register/Update the ExecNode duty list and statement;

$>$ Collect/Record executor event;

ExecNode is the resident provider to execute the business data;

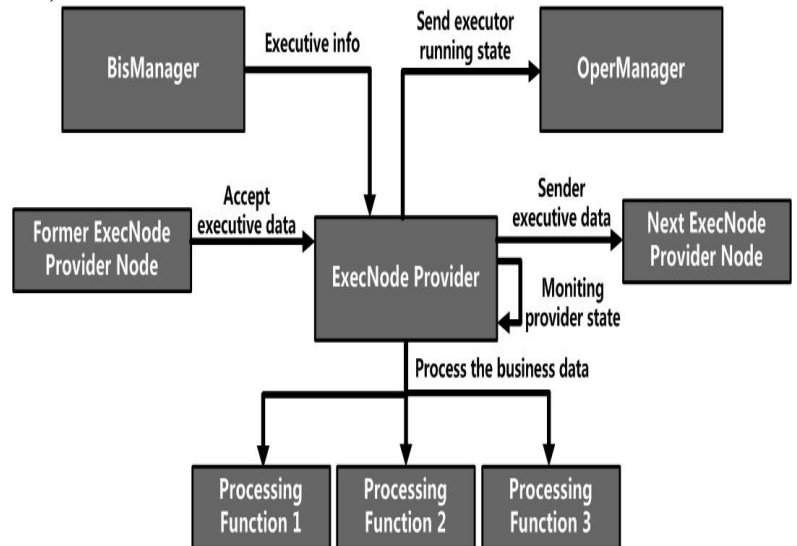

Monitor and capture the executive metadata and business data from workflow instance;

Create executive thread, the thread and workflow instance is one-on-one relationship;

Create joint connection thread to the next ExecNode;

Monitor all the threads executive statement;

\section{CONCLUSION}

The architecture fundamentally fits the need of graphics asset management system.

First of all, it's able to adapt to complex network environment. As mentioned, graphics asset management system provides the services over geographical and temporal distance. In terms of networking deployment, the system is faced with three levels of geographical environment as intranet network, metropolitan area network and internet network. The architecture can realize system hierarchical configuration in order to adapt to different geographical environments. Secondly, the architecture is designed for supporting multiple transport protocols and encapsulation data formats. HTTP, HTTPS, TCP, MSMQ as the compatible protocols and RAW, XML and JSON as business data formats are supported in current design. They can be captured, dispatched and processed to the specific executors. Finally, the performance of architecture can realize linear growth by simple online extension. It is also the fundamental concept of cloud computing mode for business.

\section{REFERENCES}

[1] Youssef Said ,All-Optical Signal Processing by Using SOA Nonlinearities: Prospects for the Evolution of Next Generation of Optical Networks[J]. Recent Patents on Signal Processing, 2012:56-62

[2] Maryam Razavian. Understanding SOA Migration Using a Conceptual Framework[J].Journal of Systems Integration,2010:3439 
[3] Vaibhav Vyas. Global SOA Architectural Style - SOAfor End Users[J].Journal of Algorithms \& Computational Technology,2010:67-71

[4] Majlesi Shahrbanoo. An Approach for Agile SOA Development using Agile Principals. [J].CoRR,2012:25-29
[5] Lutz Lowis. Vulnerability Analysis in SOA-Based Business Processes. [J].IEEE T. Services Computing,2011:45-49

[6] Latifa Boursas. Systems and Virtualization Management. Standards and the Cloud. [M].Springer Berlin Heidelberg,2010:12-19 